\title{
Erratum to: The efficacy of continuous subcostal transversus abdominis plane block for analgesia after living liver donation: a retrospective study
}

\author{
Akihiko Maeda ${ }^{1,2}$ (i) $\cdot$ Sho Carl Shibata ${ }^{2} \cdot$ Hiroshi Wada $^{3} \cdot$ Shigeru Marubashi $^{3}$ • \\ Takahiko Kamibayashi $^{2} \cdot$ Hidetoshi Eguchi $^{3} \cdot$ Yuji Fujino $^{2}$
}

Published online: 16 February 2016

(C) Japanese Society of Anesthesiologists 2016

Erratum to: J Anesth (2016) 30:39-46

DOI 10.1007/s00540-015-2085-x

The authors noticed an error in the last sentence of the second paragraph in the 'Result' section of the article, and would like to correct it as follows:

"The administration of nonopioid analgesics (Table 2), PTINR, and duration of postoperative hospital stay (Table 3) did not differ between the groups."

The online version of the original article can be found under doi:10.1007/s00540-015-2085-x.

Akihiko Maeda

amaeda@anes.med.osaka-u.ac.jp

1 Present Address: Department of Anesthesiology, National Hospital Organization Osaka National Hospital, 2-1-14

Hoenzaka, Chuo-ku, Osaka, Osaka 540-0006, Japan

2 Department of Anesthesiology and Intensive Care Medicine, Graduate School of Medicine, Osaka University, Suita, Osaka, Japan

3 Department of Gastroenterological Surgery, Graduate School of Medicine, Osaka University, Suita, Osaka, Japan 